\title{
Effect of plasma treatment on the mechanical properties of natural fiber/PP composites
}

\author{
B. S. Kim ${ }^{1}$, M. H. Nguyen ${ }^{1}$, B. S. Hwang ${ }^{1} \&$ S. Lee ${ }^{2}$ \\ ${ }^{1}$ Composite Materials Lab, Korea Institute of Materials Science, Korea \\ ${ }^{2}$ Department of Chemical Engineering, \\ Changwon National University, Korea
}

\begin{abstract}
Atmospheric glow discharge (AGD) was developed with industrial plasma sources by using high voltage radio frequency (RF) excitation at $\mathrm{KHz}$ frequency ranges. It is possible to produce a steady-state uniform glow discharge at atmospheric pressure with various gases, thus eliminating the requirement for a vacuum system to expose the materials to plasma. The RF frequency should be in the limited range to produce AGD: if it is too low, the discharge will not initiate and if it is too high, the plasma will form filamentary discharge between the electrode plates or will be transformed to the arc discharge. The advantage of AGD is to modify or coat the materials with good uniformity. The AGD plasma polymerization can be used to modify the surface properties of wood powder as well as natural fiber to improve the compatibility between the fiber surface and PP matrix. The AGD surface modification process can allow the constituents to disperse quite evenly within the matrix with a strong interfacial bonding between the constituents and the matrix material.

Contact angles of AGD polymers of various monomers were measured by goniometer and their surface energies were calculated, respectively, to find the most suitable monomer, which was hexamethyl- disiloxane. Helium was used as a carrier gas and the monomer modified the surface property by plasma polymerization. The reactor is operated at room temperature while the surface is modified at atmospheric pressure. Mechanical tests and characterizations by the SEM were carried out for the natural fiber/pp composites.
\end{abstract}

Keywords: natural fiber composites, plasma treatment, atmospheric glow discharge. 


\section{Introduction}

Over the past decade, numerous researches have been performed to develop wood powder and/or natural fiber reinforced polymers due to the low cost, renewability, biodegradability, abundancy and high specific strength and stiffness of natural fiber [1-5]. However, the compounding of wood powder or natural fiber with a polymer matrix (such as polypropylene, PP) often leads to poor mechanical properties of the composites. The poor mechanical properties of wood powder reinforced PP composites are due to (1) poor compatibility between the polar hydrophilic wood powder or natural fiber and the non-polar hydrophobic PP, with weak interfacial adhesion between wood powder or fiber and PP matrix, and (2) poor dispersion of wood powder and fiber in the PP matrix.

A new environmentally friendly method of blending wood powder and natural fiber is developed utilizing plasma treatment on the powder and the fibers, respectively. These methods utilize a non-toxic chemical bonding agent. Wood powder is dried and plasma coated in a continuous process with hexamethyl-disiloxane in a closed chamber before it is mixed with PP (Hanwha Co.) to produce pellets by an extruder. Natural fibers such as Jute and Coir are washed by $\mathrm{NaOH}$ and dried before the plasma treatments. For both cases, the plasma treatments were in a continuous process at room temperature and atmospheric pressure with no vacuum. Mechanical test results revealed the improvements.

\section{Atmospheric glow discharge}

\subsection{Selection of monomer}

Contact angles of coated polymers are measured by a goniometer. Table 1 summarizes the contact angles and calculated surface energies for various monomers. HMDSO was chosen since it is most lipophilic due to its low polar surface energy and high surface energy. The surface energies may be calculated by

$$
r_{S L}=r_{S L}+r_{L V}-2\left(r_{S}^{d} r_{L V}^{d}\right)^{1 / 2}-2\left(r_{S}^{p} r_{L V}^{p}\right)^{1 / 2} .
$$

\subsection{Plasma coating on wood powder}

As shown in Fig 1, the wood powder was dried in an atmospheric glow discharge (AGD) reactor composed of a heater. Helium was used as a carrier gas and hexamethyl-disiloxane (HMDSO) was used to modify the surface property by plasma polymerization [6]. The electrodes were inductively connected around the tube and the frequency and the voltage were $20 \mathrm{KHz}$ and $3 \mathrm{KV}$, respectively. The wood powder was plasma coated for 30 minutes. 
Table 1: $\quad$ Contact angles and calculated surface energy.

\begin{tabular}{|c|c|c|c|c|c|}
\hline \multirow{2}{*}{ Monomer liquids } & \multicolumn{2}{|c|}{ Contact angle $\left(^{\circ}\right)$} & \multicolumn{3}{c|}{ Surface energy (dyne/cm) } \\
\cline { 2 - 6 } & Water & Glycerol & Total & Dispersion & Polar \\
\hline Oxygen-PET & 8 & 9 & 73.73 & 12.78 & 60.96 \\
\hline Benzene-PET & 58.8 & 65 & 49.17 & 1.08 & 47.37 \\
\hline CH4-PET & 55.7 & 47.6 & 59.56 & 2.14 & 57.42 \\
\hline Acrylic acid-PET & 35.7 & 47.7 & 71.94 & 1.72 & 70.22 \\
\hline $\begin{array}{c}\text { Hexafluoroethane- } \\
\text { PET }\end{array}$ & 93 & 103 & 28.75 & 0.54 & 28.21 \\
\hline Trifluorotolune-PET & 49 & 53.1 & 53.81 & 4.90 & 48.91 \\
\hline HMDSO* & 105 & 76 & 74.77 & 71.68 & 3.09 \\
\hline
\end{tabular}

* HMDSO: Hexamethyl-disiloxane.

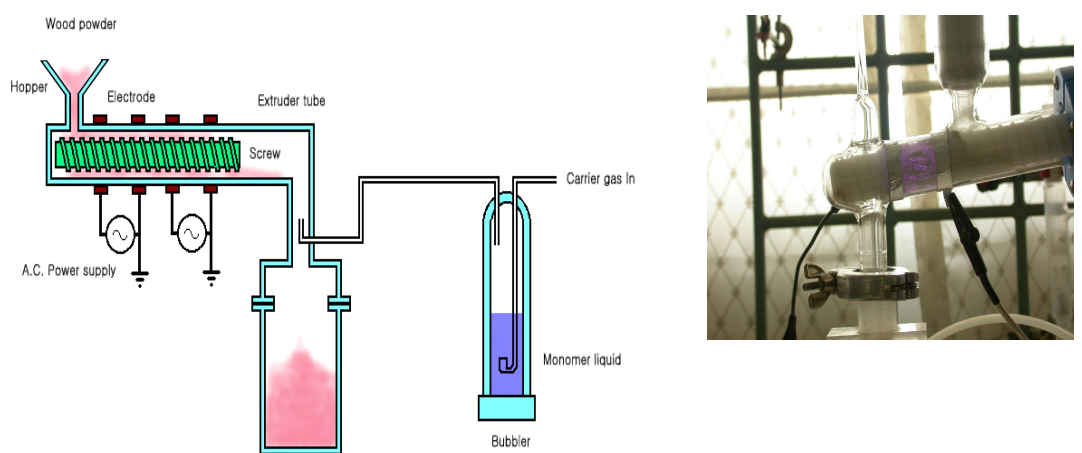

Figure 1: Continuous process of surface coating of wood powder.

\subsection{Plasma coating on natural fibers}

As shown in Fig. 2, the natural fibers are allowed to pass between the electrode plates where the plasma polymerization exists. Again, Helium was used as a carrier gas and hexamethyl-disiloxane was used to the modify surface property by plasma polymerization. The frequency and voltage were $20 \mathrm{KHz}$ and $5 \mathrm{KV}$, respectively. The fiber speed was $50 \mathrm{~mm} / \mathrm{s}$.

\section{Preparation of the specimen}

\subsection{Wood powder/PP composites}

The average moisture content in the wood powder was about $8 \%$ and this was vacuum dried to $0 \%$ moisture content before the surface treatment. The average 


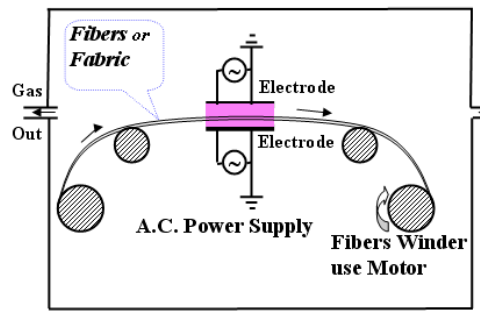

Plasma Chamber
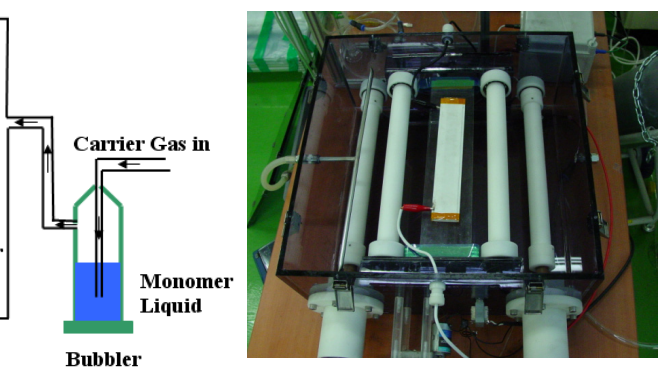

Figure 2: Continuous process of surface coating of natural fiber.

particle size was $138.22 \mu \mathrm{m}(\min =28.86 \mu \mathrm{m}, \max =346.86 \mu \mathrm{m})$ and the density was $0.40 \sim 0.42 \mathrm{~g} / \mathrm{cm}^{3}$ before drying. The size of most commercially manufactured wood powder is less than $425 \mu \mathrm{m}$.

The plasma coated wood powder was mixed with PP powder mechanically before producing pellets by PRISM TSE 16 TC (Thermo Electron Corp.) twin screw extruder (Fig. 2). The pellets are press molded to form mechanical test coupons for 10 minutes at $195^{\circ} \mathrm{C}$ and $10 \mathrm{kgf} / \mathrm{mm}^{2}$ pressure. $50 \mathrm{wt} \%$ of the wood powder was mixed with $\mathrm{PP}$, respectively, to prepare the specimen.

\subsection{Jute/PP and Coir/PP composites}

Vietnamese Jute and Coir fibers were alkali treated with 2 and $6 \% \mathrm{NaOH}$ for 24 hours, respectively. The fibers were then washed with distilled water several times to remove any traces of $\mathrm{NaOH}$ on the fiber surface, neutralized with dilute acetic acid, and again washed with distilled water. The final $\mathrm{pH}$ maintained was 7. The $\mathrm{NaOH}$ treatment on the fibers removed chemical contents such as lignin, hemi-cellulose, pectin, and changed the state of the materials from hydrophilic to hydrophobic [7]. The natural fibers were vacuum dried to zero moisture content at $75^{\circ} \mathrm{C}$ before being molded with pp fibers to form tensile test coupons. $50 \mathrm{wt} \%$ natural fibers were molded at $195^{\circ} \mathrm{C}$ and $20 \mathrm{kgf} / \mathrm{mm}^{2}$ pressure.

\section{Results and discussion}

\subsection{Wood powder composites}

Tensile tests and 3-point bending tests are carried out according to ASTM D638 (Type V) and ASTM D790, respectively. Tensile test results are shown in Fig. 3 and 3-point bending test results are shown in Fig. 4. Figure 5 shows the work done by Chungnam National University in Korea and is for comparison.

From Figs. 3 and 4, it can be noticed that the data are fairly even around the average values. This may indicate that the wood powders are quite evenly distributed within the matrix due to the enhancement of compatibility of the particles within the matrix. When comparing the data in Fig. 3 and Fig. 5, the tensile strength for $50 \mathrm{wt} \%$ wood powder composite is quite close to that of $\mathrm{PP}$ 
and is higher compared to $45 \%$ wood powder composite. This may indicate that the present method provided improved bonding between the wood flour and the PP.

The average density measured and elongation for $50 \mathrm{wt} \%$ composite were 1.1 $\mathrm{g} / \mathrm{cm}^{3}$ and $6.32 \%$ (Fig 6), respectively.

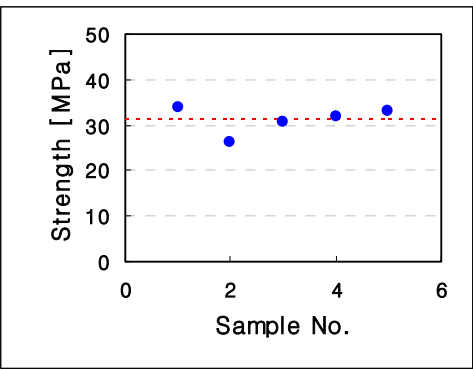

Figure 3: Tensile test results for $50 \mathrm{wt} \%$ wood powder composite.

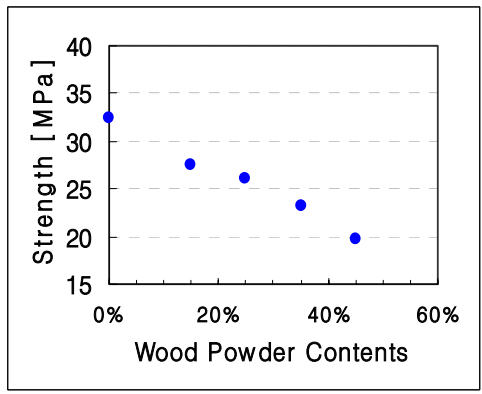

Figure 5: J. of Korea Ind. \& Eng Chem., V10, No. 1, Feb, '99.

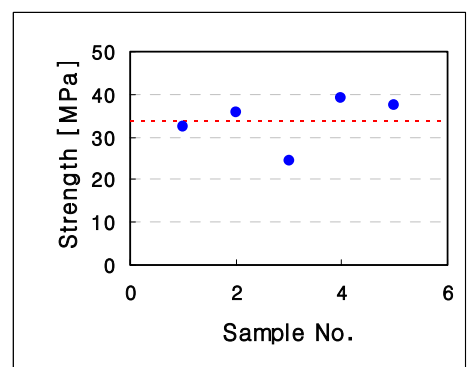

Figure 4: 3 point bending test results for $50 \mathrm{wt} \%$ wood powder composite.

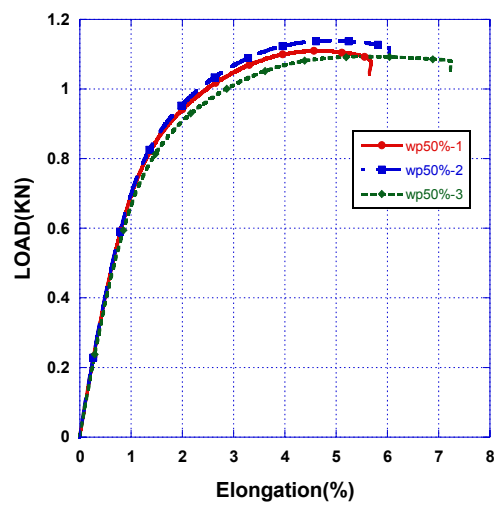

Figure 6: Elongation of 50wt $\%$ wood powder composite.

\subsection{SEM on wood powder composites}

Figure 7 represents Scanning Electron Microscopic views on the fracture surface of the $50 \mathrm{wt} \%$ wood powder/PP composite. It may be seen that the plasma surface coated wood powders are fairly evenly dispersed. 
In Figure 7, the circled regions represent wood powder. The wood powders in Fig 7(a) are dispersed quite evenly while, in (b), the wood powers are bunched up in some spots.

In Figure 8(a), the plasma treated wood powder is broken during the fracture with some PP on the wood powder and the wood powder is pulled out unharmed with no trace of PP on the pulled out portion (Fig. 8(b)).

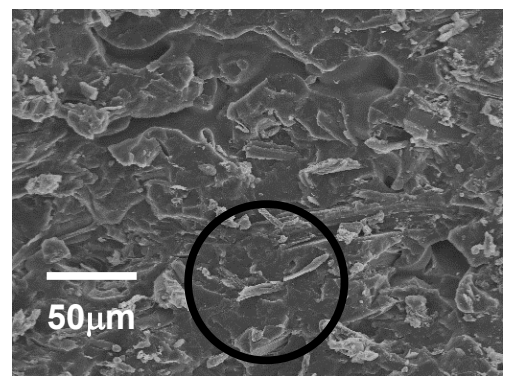

a) Plasma treated wood powder

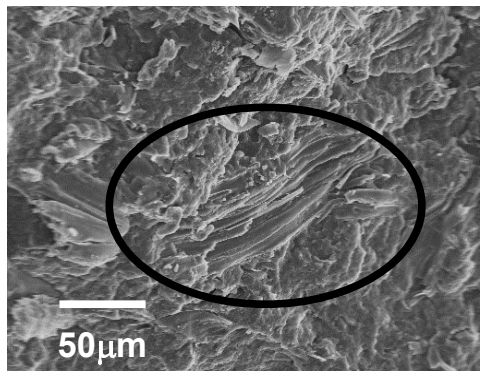

b) No treatment on wood powder

Figure 7: $\quad 50 \mathrm{wt} \%$ of wood powder within PP. The wood powder is circled.

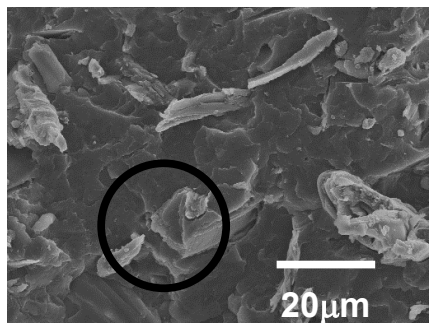

a) Plasma treated wood powder

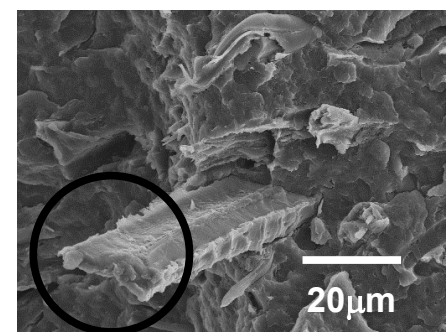

b) No treatment on wood powder

Figure 8: $\quad 50 \mathrm{wt} \%$ of wood powder within PP. The pulled out wood powder is circled.

\subsection{Jute/PP and Coir/PP composites}

Tensile strength and elongation were tested according to ASTM - D 638 Type I. For each mechanical test, a minimum of 10 specimens were tested. In Figs. 9 and 10, the average tensile strengths and elongations for Jute/PP and Coir/PP composites are shown, respectively. For Jute/PP composites, the plasma treatment increased tensile strength by 50 and $114 \%$, respectively, compared with $\mathrm{NaOH}$ treated and raw fibers. As for Coir/PP composites, the plasma treatment increased tensile strength by 22 and $92 \%$, respectively, compared with $\mathrm{NaOH}$ treated and raw fibers. 


\subsection{SEM on natural fiber composites}

Figure 11 represents Scanning Electron Microscopic views on the fracture surface of the 50wt \% Coir/PP composite. Figure 11(a) shows the poor adhesion (circle) between the fibers and PP. Figure 11(b) shows some PP on the fibers and Figure 11(c) shows much more PP on the pulled out fibers. This may indicate that the best adhesion was obtained from the plasma treatment on the Coir fibers. This result is also verified by the tensile test results.

Scanning Electron Microscopic views on the fracture surface of the $50 \mathrm{wt} \%$ Jute/PP composite were not taken since the fracture surface was too rough to take SEM.
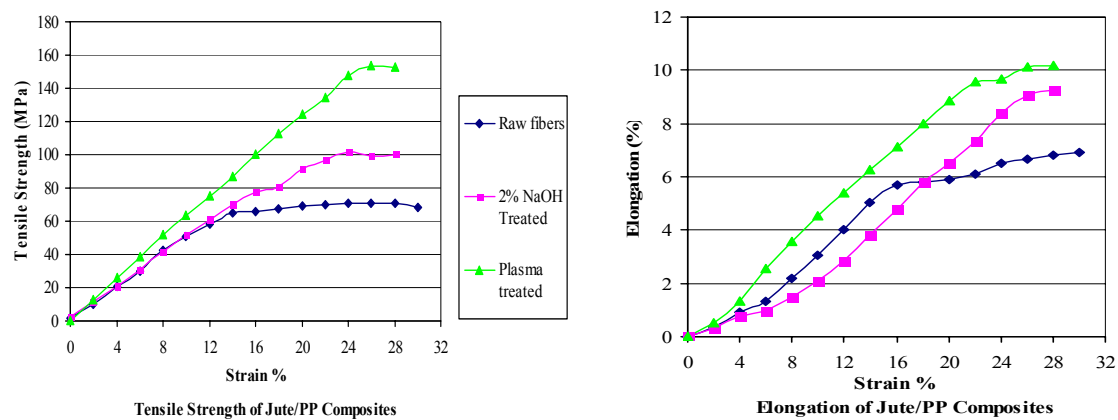

Figure 9: $\quad$ Tensile test results and elongations for 50wt\% Jute/PP composite.

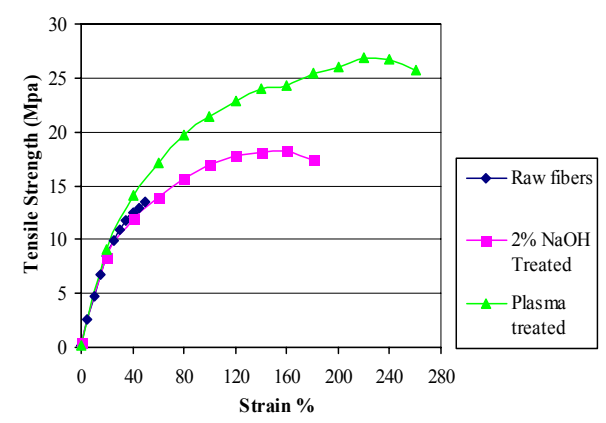

Tensile strength of Coir/PP Composites

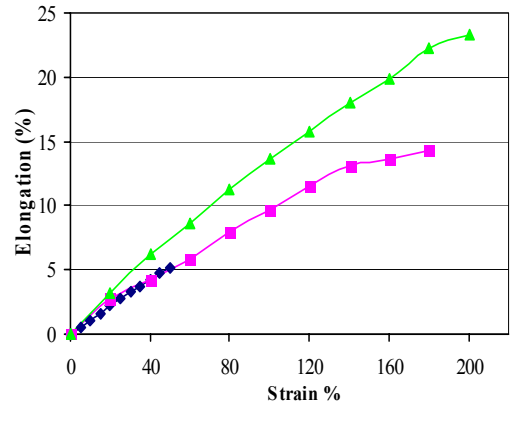

Elongation of Coir/PP composite

Figure 10: Tensile test results and elongations for $50 \mathrm{wt} \%$ Coir/PP composite.

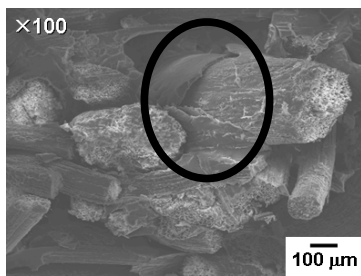

(a) No treatment on fibers

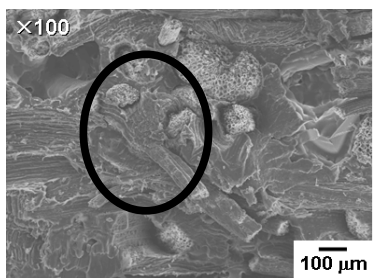

(b) $6 \% \mathrm{NaOH}$ treatment

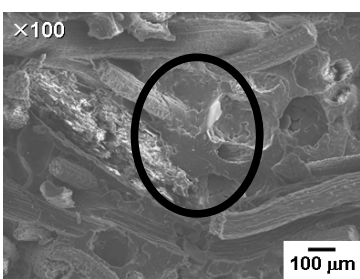

(c) Plasma treatment

Figure 11: SEM of 50\% Jute fiber/PP composites. 
Densities of raw Jute and Coir fibers were 1.15 and $1.25 \mathrm{~g} / \mathrm{cm}^{3}$, respectively. The densities of the plasma treated Jute/PP and Coir/PP were 1.029 and 1.068 $\mathrm{g} / \mathrm{cm}^{3}$, respectively.

\section{Conclusions}

- Non-toxic surface treatment methodology was introduced. Hexamethyldisiloxane was used to plasma coat the wood powder and natural fibers were used to improve its bonding and dispersion with the polypropylene. The good compatibility between the wood powder and the PP lead to relatively good interfacial adhesion and dispersion between the wood powder and the PP matrix.

- The mechanical test results and SEM inspections also indicate that the wood powder was quite evenly dispersed within the PP matrix and has good interfacial bonding with PP. This may be due to the enhancement of the compatibility of the wood powder within the PP matrix.

- For the Jute/PP and Coir/PP composites, the $\mathrm{NaOH}$ and plasma treatments on the fibers have clearly improved the tensile strengths.

\section{References}

[1] M Defosse, Wood Composites are Expanding among Sectors, MODERN PLASTICS, Jan. 2003, P30-33

[2] L. Czarnecki and J. L. White, J of Appl. Polym. Sci, 25(1980) 1217.

[3] H. Dalvag, C. Klason and H. E. Stromvall, Int. J. Polym Mater., 11(1985) 9

[4] A. J. Michell, Appita 39 (1986) 223

[5] B. S. Sanschagrin, T. Sean and B. V. Kotta, J. of Thermoplast. Comps. Mater. 1(1988) 184

[6] H. Yasuda, Plasma Polymerization, Academic Press, Orlando, Florida (1985)

[7] K. W. Sellers, R. W. Zeigler IV and S. L. Morgan, Forensic Discrimination of selected Vegetable Fibers by Pyrolysis-Gas Chromatography/ Mass Spectrometry and Multivariate Statistics, Dept. of Chemistry and Biochemistry, The U. of Carolina-Columbia, SC, 29028. 2003. 